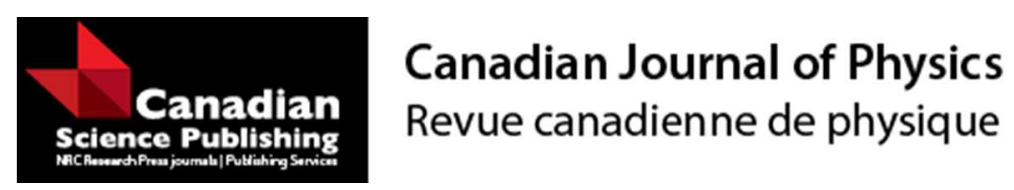

\title{
Electric dipole transition probabilities, oscillator strengths and lifetimes for $\mathrm{Co}^{16+}$
}

\begin{tabular}{|r|l|}
\hline Journal: & Canadian Journal of Physics \\
\hline Manuscript ID & cjp-2015-0414.R1 \\
\hline Manuscript Type: & Article \\
\hline Complete List of Authors: & $\begin{array}{l}\text { Çelik, G.; Selcuk University, Physics } \\
\text { Ateş, Ş.; Selcuk University, Physics } \\
\text { Tekeli, G.; Selcuk University, Physics }\end{array}$ \\
\hline Keyword: & $\begin{array}{l}\text { electric dipole transition, transition probabilities, oscillator strengths, } \\
\text { WBEPM theory, Co16+ }\end{array}$ \\
\hline \multicolumn{2}{|c}{} \\
\hline
\end{tabular}


Electric dipole transition probabilities, oscillator strengths and lifetimes for $\mathrm{Co}^{16+}$

\author{
G. Çelik ${ }^{1}$, Ş. Ateş, and G. Tekeli \\ Department of Physics, Faculty of Science, Selçuk University, Campus 42049 Konya, Turkey
}

\begin{abstract}
The electric dipole transition probabilities, oscillator strengths and lifetimes for $\mathrm{Co}^{16+}$ have been calculated within the weakest bound electron potential model (WBEPM) theory using experimental energy levels and theoretical expectation values of orbital radii corresponding to those energy levels under the assumption of the $L S$ coupling scheme. In the calculations both multiplet and fine structure transitions are studied. The present results are consistent with earlier results given in the literature. Moreover, some transition probability and oscillator strength values not existing in the literature are reported for the first time.
\end{abstract}

PACS No: 32.70 . Cs

Corresponding author, e-mail: gultekin@selcuk.edu.tr 


\section{Introduction}

Studies on highly ionized atoms are an attractive subject in many fields such as astrophysics, plasma physics and space research. Also, these atoms provide a quite sensitive measure of the reliability of approximate theoretical procedures [1,2]. Especially, spectra of sodium-like ions to diagnose the hot gases found in Tokamaks, laser-produced plasmas and other devices for controlled-fusion research are used [3,4] and a knowledge of the properties related to these spectra is important for detecting the presence of sodium-like ions in the plasmas and for calibrating spectral measurements $[4,5]$. Since the energy levels of highly charged sodium-like ions are practically free from effects of configuration mixing, it can be said that spectra of these ions have a simple structure. Therefore, they are well suited for diagnostic purposes and for a theoretical interpretation of line intensities [6]. $\mathrm{Co}^{16+}$ is one of the sodium-like ions that they have one electron outside a closed shell. Ionized cobalt is very important in astrophysics, and also as impurities in magnetic fusion plasmas. A number of lines of cobalt have been identified in astrophysical studies [7].

Up to now, transition probabilities and oscillator strengths for $\mathrm{Co}^{16+}$ have been the subject of many theoretical and experimental studies. Mc Eachran et al. [8] calculated the oscillator strengths using the frozen-core orbital wave-functions for all permitted electric dipole transitions between the various states in Fe XVI, Co XVII and Ni XVIII. Tull et al. [9] calculated the dipole oscillator strengths with the frozen-core Hartree-Fock approximation for allowed transitions between some levels with non-relativistic ionization energies. Martin et al. [4] presented the oscillator strengths for some transitions in sodium-like atoms with the quantum defect orbital (QDO) and its relativistic (RQDO) counterpart. Fuhr et al. [10] compiled the theoretical transition probabilities, oscillator strengths and line strengths for allowed spectral lines of the elements iron, cobalt, and nickel in all stages of ionization. Lavin et al. [2] reported the oscillator strengths of the principal spectral series of several members of the sodium isoelectronic sequence with the QDO method and its relativistic counterpart. Fine-structure energy levels, oscillator strengths and transition probabilities for transitions among the terms belonging to some configurations were calculated using configuration interaction code (CIV3) by Younis et al. [6]. Smith and Wiese [11] presented the oscillator strengths derived by interpolation for $\mathrm{Co}^{16+}$. Wavelengths, energy levels, level classifications, oscillator strengths and atomic transition probabilities for the cobalt ions from Co VIII to Co XXVII were tabulated by Shirai et al. [12]. Their results contain experimental measurements and observations. Theodosiou and Curtis [13] reported the $f$ values using the existing data base and Dirac-Fock methods in the sodium isoelectronic series. Liaw [14] computed the oscillator strengths and transition amplitudes on the frozen-core Dirac-Fock approximation using the B-spline method. 
Weiss [15] calculated the line strengths for the lowest $\Delta n=0$ transitions of ions in the lithium, sodium and copper isoelectronic sequences using Hartree-Fock method.

In this study, the transition probabilities, oscillator strengths and lifetimes for all allowed transitions between states with $n \leq 10$ and $l \leq 4$ have been generated. The transitions between states with $n \leq 5$ and $l \leq 4$ might be useful in astrophysical applications, thermonuclear fusion research and plasma diagnostics. The ones except for these states have been considered to compose data for $\mathrm{Co}^{+16}$. The calculations have been done for both multiplets and individual lines.

\section{Theory and calculation procedure}

The weakest bound electron potential model (WBEPM) theory as a model potential has been development to describe the electronic motion in a multi-electron system by Zheng [16,19]. It has been applied to calculate various atomic properties such as energy levels, ionization potentials, transition probabilities, oscillator strengths and lifetimes of excited levels in the many electron atomic and ionic systems. A complete description of the various formulations of the WBEPM theory has been presented in previous papers [16-25]. We summarize here the most important parts of the approximation. This approximation is based on the idea that electrons in a system can be divided into two groups of ones to be the weakest bound electron (WBE) and the non-weakest bound electrons (NWBE). The WBE in a given many-electron system is the most weakly bound electron to the system in comparison to the other electrons in the system. The principal quantum number $n^{*}$ and the angular momentum quantum number $l^{*}$ of the WBE are replaced by the effective principal quantum number $n^{*}$ and the effective angular momentum quantum number $l^{*}$.

According to the WBEPM theory, the Schrödinger equation of WBE under non-relativistic conditions (in a.u.) is given as [16-19],

$$
\begin{gathered}
{\left[-\frac{1}{2} \nabla^{2}+V\left(r_{i}\right)\right] \psi_{i}=\varepsilon_{i} \psi_{i}} \\
V\left(r_{i}\right)=-\frac{Z^{*}}{r_{i}}+\frac{[d(d+1)+2 d \ell]}{2 r_{i}^{2}}
\end{gathered}
$$

In this approximation the WBE moves in the potential field produced by the nucleus and the NWBE. This potential field can be divided into two parts, one of which is the Coulomb potential. Because of the orbital penetration effect of the WBE, shielding by the NWBE is not complete. 
Therefore, an effective nuclear charge $Z^{*}$ is used in the coulomb term of the potential function in the WBEPM theory. The second part is the dipole potential. Because the WBE polarizes the atomic core, a dipole moment is produced.

In equation (2), $V\left(r_{i}\right)$ is potential function produced by the NWBE and nucleus. Moreover, $\langle r\rangle$ is the distance between the $i \mathrm{WBE}$ and nucleus. In this theory, electronic radial wave functions are presented as a function of Laguerre polynomial in terms of some parameters which are determined using the experimental energy data and the expectation values of radii $[20,21]$.

The radial wave function of the WBE can be obtained to be

$$
R_{n^{*} l^{*}}(r)=\left(\frac{2 Z^{*}}{n^{*}}\right)^{l^{*}+3 / 2}\left[\frac{2 n^{*}}{(n-l-1) !} \Gamma\left(n^{*}+l^{*}+1\right)\right]^{-1 / 2} \exp \left(-\frac{Z^{*} r}{n^{*}}\right) l^{l^{*}} L_{n-l-1}^{2 l^{*}+1}\left(\frac{2 Z^{*} r}{n^{*}}\right)
$$

Where $\Gamma$ is the gamma function and $L_{n-l-1}^{2 l^{*}+1}\left(\frac{2 Z^{*} r}{n^{*}}\right)$ is the general Laguerre polynomial. The $Z^{*}, n *$ and $l *$ parameters are obtained by solving the following couple of equations:

$$
I=-\varepsilon=\frac{Z *^{2}}{2 n *^{2}} \quad\langle r\rangle=\frac{3 n^{2}-\ell *(\ell *+1)}{2 Z *}
$$

Here $I$ is ionization energy and $\langle r\rangle$ is expectation values for radius of the WBE. In the present article, we present the results of calculation of the E1 transition probabilities, oscillator strengths and lifetimes for both multiplet and individual lines belonging to excited levels of $\mathrm{Co}^{16+}$ by using the WBEPM theory. In the calculations, many of transition arrays are considered.

\section{Results and Discussion}

In the WBEPM theory, the $Z^{*}, n^{*}, l^{*}$ parameters are required for atomic structure calculations. The experimental energy values and the expectation values of radii belong to the states are used to obtain these parameters. In this work, the majority of the required energy values have been taken from NIST [26]. Also, the energy values are taken from ref. [9] for 6s-10s, 7p-10p, 10d and 9f, 10f levels and from ref. [27] and ref. [28] for 8d, 9d levels not existing in NIST database. We presented already in previous papers in details how to obtain these parameters [21-23, 29-31]. 
In Table 1, the electric dipole transition probabilities and the oscillator strengths among multiplet lines are listed (Table 1 is given in the supplementary material of this paper). Many of the oscillator strengths are compared with the accepted values in NIST [26], the values determined by Tull et al. [9] and the interpolation results given by Smith and Wiese [11] for some transitions. As a result of these comparisons, we can say that our oscillator strength values are very close to the experimental and theoretical values compiled by NIST. The average disagreement of oscillator strength values obtained using the WBEPM theory is within $\pm 2.9 \%$ except for $3 p-4$ s transitions to NIST, within $\pm 2 \%$ to results obtained by Tull et al., within $\pm 10 \%$ to results given by Smith and Wiese. For $3 p-4 s$ transitions, there are some discrepancies between our results and values given in the NIST. The estimated uncertainties of accepted values in the NIST are \pm 25 for $3 p-4 s$ transitions. However, our results are more agreement with the results given in refs. [9] and [11] for these transitions.

We have listed the transition probabilities and the oscillator strengths between fine structure lines for $\mathrm{Co}^{16+}$ in Table 2 (Table 2 is given in the supplementary material of this paper). Our oscillator strength results are compared with the accepted values in NIST [26], the results tabulated by Shirai et al. [12], critically compiled results given by Fuhr et al. [10] and the results given by Younis et al. [6]. Moreover, for a few transitions some comparisons have been done with the results obtained by using QDO and RQDO theory by Lavin et al. [2], the results given by Theodosiou and Curtis [13] and the frozen-core Dirac-Fock approximation results using the B-spline method given by Liaw [14]. As a result of these comparisons the average disagreement of oscillator strength values obtained with the WBEPM theory is within $\pm 2.6 \%$ except for $3 \mathrm{p}-4 \mathrm{~s}$ transitions to NIST, within $\pm 2,8 \%$ to results tabulated by Shirai et al. and within $\pm 2,5 \%$ to results obtained by Fuhr et al. [10]. The average agreement between our results and other results given in Ref. [2, 13,14] are less than $\pm 3 \%$. The accuracy rates of accepted values given in the NIST database are within $\% 10$ 25. Therefore, our oscillator strength values are in good agreement both with the accepted values compiled by NIST database and with other literature data. The results given by Ref. [6] are not in agreement with our results and other results given in Table 2 . The inconsistency between oscillator strength values given by Ref. [6] and other results in the Table 2 are over $\pm 70 \%$.

The radiative lifetimes give some information about atomic structure. These information extracted from atomic lifetime calculations and measurements can be of relevance to a wide variety of areas from astrophysics to lamp design. For this reason, the radiative lifetimes for $\mathrm{Co}^{16+}$ are computed. Lifetimes of excited states determined from the transition probability values are given in Table 3. 


\section{Conclusions}

The overall agreement of our calculations with other results can be considered to be reasonable. In this study, previously unpublished transition probabilities, oscillator strengths and lifetimes have been obtained for $\mathrm{Co}^{16+}$. We have concluded that the semi-empirical method such as the WBEPM theory that needs the existing experimental data for adjusting one and more parameters can be considered as a useful method for highly ionized systems. The WBEPM theory is one of the simple, reliable and rapid computational methods. However, the usefulness and reliability of the WBEPM theory can be discussed by making further calculations and comparisons.

Relativistic effects on the energy levels are by no means negligible in the highly ionized members of the sodium iso-electronic sequence. The relativistic and the configuration interaction effects are considered partially through the use of the energy and the expectation values used in determination of required parameters in the WBEPM theory. Accurate estimates of radiative transition probabilities among multiplet states for successful identification of the spectra of astrophysical and laboratory plasma are an important source. The transition probabilities, the oscillator strengths and lifetimes obtained from this study can be useful in analyzing older experiments and planning new ones. At the same time, the results obtained from the WBEPM theory can serve as comparison data for theoretical and experimental studies in atomic physics.

Acknowledgment The authors gratefully acknowledge the Selçuk University Scientific Research Projects (BAP) Coordinating Office for support.

\section{References}

1. A.K. Pradhan. Phys. Scr. 35, 840 (1987).

2. C. Lavin, I. Martin, J. Karwowski. J. Mol. Struc. (Theochem), 86, 161 (1992).

3. N.J. Peacock, M.F. Stamp, J.D. Silver. Phys. Scr. T8, 10 (1984).

4. I. Martin, J. Karwowski, C. Lavin, G.H.F. Diercksen. Phys. Scr. 44, 567 (1991).

5. J. Reader et al. J. Opt. Soc. Am. 4, 1821 (1987).

6. W.O. Younis, S.H. Allam, Th. M. El-Sherbini. At. Data Nucl. Data Tables, 92, 187 (2006).

7. E. Charro, I. Martin. Astron. Astrophys. 376, 1106 (2001).

8. R.P. Mc Eachran, C.E Tull, M. Cohen. Astron. Astrophys. 4, 152 (1970).

9. C.E. Tull, R.P. Mc Eachran, M. Cohen. Atomic Data, 3, 169 (1971). 
10. J.R. Fuhr, G.A. Martin, W.L. Wiese, S.M. Younger. J. Phys. Chem. Ref. Data, 10, 305 (1981).

11. M.W. Smith, W.L. Wiese. Astrophys. J. Suppl. Ser. 196, 103 (1971).

12. T. Shirai et al. J. Phys. Chem. Ref. Data, 21, 23 (1992).

13. C.E. Theodosiou, L.J. Curtis. Phys. Rev. A, 38, 4435 (1988).

14. S. Liaw. Can. J. Phys. 70, 644 (1992).

15. A.W. Weiss. J. Quant. Spectrosc. Radiat. Transfer, 18, 481 (1977).

16. N.W. Zheng. A new outline of atomic theory. Jiang su education press nanjing PR China (1988).

17. N.W. Zheng, T. Wang, R.Y. Yang. J. Chem. Phys. 113, 6169 (2000).

18. N.W. Zheng, T. Wang, D.X. Ma, T. Zhou, J. Fan. Int. J. Quant. Chem. 98, 281 (2004).

19. N.W. Zheng et al. At. Data Nucl. Data Tabl. 79, 109 (2001).

20. N.W. Zheng, T. Wang. Astrophys. J. Supp. Ser. 143, 231 (2002).

21. G. Çelik, Ş. Ateş, S. Özarslan, M. Taşer. J. Quant. Spectrosc. Radiat. Transfer, 112, 2330 (2011).

22. G. Çelik, E. Erol, M. Taşer. J. Quant. Spectrosc. Radiat. Transfer, 129, 263 (2013).

23. G. Çelik, D. Doğan, Ş. Ateş, M. Taşer. J. Quant. Spectrosc. Radiat. Transfer, 113, 1601 (2012).

24. N.W. Zheng, T. Wang, D.X. Ma, T. Zhou, J. Fan. Int. J. Quant. Chem. 98, 281 (2004).

25. N.W. Zheng et al. J. Phys. Soc. of Japan, 68, 3859 (1999).

26. A. Kramida, Yu Ralchenko, J. Reader NIST ASD Team, 2013, NIST Atomic Spectra Database (ver. 5.1), [Online]. Available: http://physics.nist.gov/asd National Institute of Standards and Technology, Gaithersburg, MD.

27. H. Ray. J. Phys. B: At. Mol. Opt. Phys. 35, L299 (2002).

28. H. Ray. Astron. Astrophys. 391, 1173 (2002).

29. G. Çelik, Ş. Ateş. Can. J. Phys. 86, 487 (2008).

30. I. K. Ozturk, G. Çelik, Y. Gokce, et al. Can. J. Phys. 92, 1425 (2014).

31. Ş. Ateş, Y. Gökçe, G. Çelik, M. Yıldız. Can. J. Phys. 92, 1043 (2014). 